\title{
HOMOLOGY AND CELL STRUCTURE OF NILPOTENT SPACES
}

\author{
BY
}

\author{
ROBERT H. LEWIS
}

\begin{abstract}
Let $A$ and $X$ denote finitely dominated nilpotent $\mathrm{CW}$ complexes. We are interested in questions relating the homology groups of such spaces to their cell structure and homotopy type. We solve a problem posed by Brown and Kahn, that of constructing nilpotent complexes of minimal dimension. When the fundamental group is finite, the three-dimensional complex we construct may not be finite; we then construct a finite six-dimensional complex.

We investigate the set of possible cofibers of maps $A \rightarrow X$, and find a severe restriction. When it is met and the fundamental group is finite, $X$ can be constructed from $A$ by attaching cells in a natural way. The restriction implies that the classical notion of homology decomposition has no application to nilpotent complexes.

We show that the Euler characteristic of $X$ must be zero. Several corollaries are derived to the theory of finitely dominated nilpotent complexes.

Several of these results depend upon a purely algebraic theorem that we prove concerning the vanishing of homology of nilpotent modules over nilpotent groups.
\end{abstract}

0. Introduction. The topological concept of nilpotency is a generalization of that of simple connectivity. Since nilpotent complexes satisfy the Whitehead Theorem, one would like to derive their homotopy type by starting with their homology groups. We are therefore interested in the following sort of questions, all of which have affirmative or easy answers for simply connected complexes.

Given nilpotent complexes $A$ and $X$ with the same finitely generated fundamental group $\pi$ :

(1) If all ordinary cohomology groups $H^{i}(X, G)$ vanish for all Abelian groups $G$ and for all $i>n$, can $X$ be represented by an $n$-dimensional complex?

(2) If $X$ has only finitely many nonvanishing homology groups, all finitely generated, is $X$ finitely dominated? finite?

(3) Given a finite sequence of finitely generated Abelian groups, is there a nilpotent space whose homology is that sequence? (There would be obvious restrictions at the lower end.)

(4) Can any homotopy type appear as the cofiber of a map $A \rightarrow X$ ? (If the map is an isomorphism on fundamental groups, the cofiber would be simply connected.)

(5) What are the smallest and simplest nilpotent complexes?

(6) Is there a structure theorem whereby one can construct the homotopy type of $X$ from its homology groups and other algebraic invariants?

Received by the editors January 5, 1984 and, in revised form, June 1, 1984 and October 24, 1984. 1980 Matheinatics Subject Classification. Primary 55N25; Secondary 57-04, 57M99, 20C05, 20 C07. 
Question (1) was answered in the affirmative by Brown and Kahn [2]. For question (2), Mislin has shown that such an $X$ is finitely dominated but not necessarily finite $[10,11]$. The first five questions may be thought of as steps toward understanding the sixth.

In this paper we contribute to questions (3)-(6).

In $\S 1$ we establish a purely algebraic result, Theorem A, here summarized:

THEOREM A. Suppose that $\pi$ is a finitely generated nilpotent group and that $M$ is a nonzero nilpotent $Z \pi$-module with $H_{n}(\pi, M)=0$, some $n \geqslant 0$. If $\pi$ is finite and a modest condition on $M$ holds, then $M$ has to be finite and cohomologically trivial. If $\pi$ is infinite, then $n>\operatorname{rank}(\pi)$.

In $\$ 2$ we show that the Euler characteristic of a nonsimply-connected finitely dominated nilpotent complex is zero. (Part of this result was mentioned in paper by Mislin [12].) Therefore, the answer to question (3) above is no for any nontrivial fundamental group. Several other applications are derived.

$\$ 3$ answers question (4) above negatively. We consider maps that are almost homology equivalences in the sense that the cofiber $X / A$ is a Moore space, i.e., a simply-connected space $K^{\prime}(G, n)$ with one nonvanishing homology group $G$ in dimension $n$. We prove

THEOREM B. Let $A \rightarrow X$ be a cofibration of nilpotent complexes with cofiber $X / A=K^{\prime}(G, n), n \geqslant 3$. Let $\pi=\pi_{1} X \neq 0$.

(1) If $\pi$ is infinite but finitely generated, then $G=0$.

(2) If $\pi$ is finite and $H_{n}(\tilde{X}, \tilde{A})$ is finitely generated, then $G$ must be finite and $\tilde{X} / \tilde{A}$ is a Moore space.

Further, $X$ can be built from $A$ by attaching $n$-and $(n+1)$-dimensional cells.

The following result, relevant to question (6) above, shows that the classical concept of homology decomposition [7] does not apply to nilpotent spaces.

THEOREM C. If a finitely dominated nilpotent complex has a homology decomposition of the classic sort, then either it is simply connected or the decomposition is trivial.

$\S 4$ is concerned with question (5). In [2] Brown and Kahn prove a lower bound on the dimension of a nilpotent complex having fundamental group $\pi$. They pose the problem: when is this lower bound met?

THEOREM D. For any finitely generated nilpotent group $\pi$ the minimal dimension is realized by a finitely dominated nilpotent complex $X$. Further:

(1) If $\pi$ is torsion free, the universal cover $\tilde{X}$ is an equivariant Moore space of type $(M, 2, \pi)$, where $M$ is $Z$-free and nilpotent.

(2) If $\pi$ is infinite, $X$ may be taken finite.

(3) For the case $\pi$ finite (for which the minimal dimension is three), if $\pi$ is cyclic, or is a p-group, $X$ may be taken finite. For any finite $\pi$ there is a finite six-dimensional nilpotent complex. 
We now summarize some definitions and known results. A $\pi$-module is a left $Z \pi$-module. $I \subset Z \pi$ is the augmentation ideal. A $\pi$-module $M$ is said to be prenilpotent if the tower $M \supset I M \supset I^{2} M \supset \cdots$ stabilizes at some point $\left(I^{n} M=\right.$ $\left.I^{n+1} M\right)$, nilpotent if it reaches 0 , perfect if $I M=M$. Over finitely generated nilpotent $\pi$, perfect modules form a Serre class [8]. So do prenilpotent modules [op. cit.]. A prenilpotent space is one whose homology modules $H_{*} \tilde{X}$ are prenilpotent. For finite $\pi, M$ is said to be cohomologically trivial if all Tate cohomology groups, over all subgroups of $\pi$, vanish (cf. Rim [16]). If $\pi$ is cyclic, generated by $x$, there is a particularly nice $\pi$-resolution of $Z$. For $\pi=Z_{n}(\equiv Z / n Z$ in this paper $)$ it is

$$
\cdots \stackrel{T}{\rightarrow} Z \pi \stackrel{N}{\rightarrow} Z \pi \stackrel{T}{\rightarrow} Z \pi \rightarrow Z \rightarrow 0
$$

where $T(w)=(x-1) w$ and $N(w)=\left(1+x+x^{2}+\cdots+x^{n-1}\right) w$. For $\pi$ infinite cyclic $T$ is an injection. This resolution can be realized geometrically by a $K(\pi, 1)$. That is, one can build a $K(\pi, 1) X$ so that $H_{n}\left(\tilde{X}^{n}, \tilde{X}^{n-1}\right)=Z \pi$ and have boundary maps alternately $T$ and $N$.

A nilpotent space is finitely dominated iff it has finitely many nontrivial homology groups, each finitely generated [11, p. 547]. Any covering of a finitely dominated nilpotent space is finitely dominated and nilpotent [11, p. 549].

I am indebted to Peter Kahn, Ken Brown and Armand Brumer for many helpful conversations.

1. Vanishing homology over nilpotent groups. The main result of this section concerns the vanishing of homology over nilpotent groups. It is similar in spirit to results of Rim [16], Dwyer [4], and Robinson [15]. The theorem says, in effect, that it is unusual for a nilpotent module over a nilpotent group to have any vanishing homology groups.

TheOREM A. Suppose that $\pi$ is a finitely generated nilpotent group and that $M$ is a nonzero nilpotent $Z \pi$-module with $H_{n}(\pi, M)=0$, some $n \geqslant 0$. Suppose, further, that one of the following holds:

(a) $\pi$ finite, $M$ finite.

(b) $\pi$ finite, $H_{n+1}(\pi, M)=0$.

(c) $\pi$ finite, $M$ finitely generated, and $n=1$.

(d) $\pi$ not finite.

Then all of the following hold:

(a) $n>\operatorname{rank}(\pi)$.

(b) $H_{j}(\pi, M)=0$ for all $j>\operatorname{rank}(\pi)$.

(c) If $\pi$ is finite and nontrivial, then $M$ is finite and cohomologically trivial.

Proof. The proof is accomplished via Propositions 1.1, 1.3, 1.4, 1.6 and 1.7.

Proposition 1.1. Let $\pi$ be a finite nilpotent group and $M$ a finite $\pi$-module with $H_{n}(\pi, M)=0$ for some $n \geqslant 1$. Then $H_{i}(\pi, M)=0$ for all $i \geqslant 1$.

Proof. This was discovered by Hoechsmann, Roquette and Zassenhaus [6]. We include a proof here because it is archetypical of most of the proofs in this section.

The main step in the proof is the following lemma. 
Lemma 1.2. Proposition 1.1 is true if $\pi$ and $M$ are both p-groups.

Proof. First observe that Proposition 1.1 is true if $\pi$ is cyclic: Using the $\pi$-resolution $(0.1)$ we have $|M|=|\operatorname{ker} N||\operatorname{im} N|=|\operatorname{ker} T||\operatorname{im} T|$, so all homology groups have the same number of elements.

It is well known that if $\pi$ and $M$ are both finite $p$-groups, the action of $\pi$ on $M$ must be nilpotent.

For the time being assume that $n=1$. We shall prove this restricted version of the lemma by induction on $|\pi|$.

Let $\alpha$ be any nontrivial normal subgroup of $\pi$. Look at the Lyndon-HochschildSerre spectral sequence $E_{s t}^{2}=H_{s}\left(\pi / \alpha, H_{t}(\alpha, M)\right) \Rightarrow H_{s+t}(\pi, M) . H_{t}(\alpha, M)$ is a nilpotent $\pi / \alpha$-module. $H_{1}(\pi, M)=0$ forces $E_{10}^{\infty}=0=E_{01}^{\infty}$. But clearly $E_{10}^{\infty}=E_{10}^{2}$ $=H_{1}\left(\pi / \alpha, H_{0}(\alpha, M)\right)$. Since the lemma is true for $\pi / \alpha, H_{s}\left(\pi / \alpha, H_{0}(\alpha, M)\right)=0$ for all $s \geqslant 1$.

In particular $E_{20}^{2}=0$ so that $E_{01}^{\infty}=E_{01}^{2}$. Thus $H_{0}\left(\pi / \alpha, H_{1}(\alpha, M)\right)=0$. This means that $H_{1}(\alpha, M)$ is both perfect and nilpotent, so must be 0 . Since the lemma is true for $\alpha, H_{t}(\alpha, M)=0$ for all $t \geqslant 1$. Therefore the entire $E^{2}$ term collapses and the result follows.

We now prove the full lemma by induction on $n$. If $M$ is a finite $\pi$-module with $H_{n}(\pi, M)=0$, map the induced module $Z \pi \otimes M$ onto $M$ by $x \otimes m \rightarrow x \cdot m$. The kernel is a finite $p$-group and has vanishing homology in dimension $n-1$. Therefore $H_{i}(\pi, M)=0$ for all $i \geqslant 2$. By Rim's theorems [16] $H_{1}(\pi, M)$ must vanish also.

We can now prove Proposition 1.1. Decompose $M$ as a direct sum of $p$-groups, $M=\oplus M_{i}$. Then certainly $H_{k}(\pi, M)=0$ iff every $H_{k}\left(\pi, M_{i}\right)=0$, so we may assume in the sequel that $M$ is a $p$-group.

If $M$ is a $p$-group and $\pi$ is a finite group consisting of torsion prime to $p$, then it is well known that $H_{i}(\pi, M)=0$ if $i \geqslant 1$. Further, $H_{0}(\alpha, M)$ is a $p$-group no matter what $\alpha$ is.

So assume now that $M$ is a $p$-group and $\pi$ is a finite nilpotent group. Let $\alpha=$ the product of all the Sylow $q$-groups of $\pi$ for $q \neq p$. Apply the elementary facts of the preceding paragraph to the spectral sequence $E_{s t}^{2}=H_{s}\left(\pi / \alpha, H_{t}(\alpha, M)\right) \Rightarrow$ $H_{s+t}(\pi, M)$ and deduce that $H_{k}(\pi, M) \cong H_{k}\left(\pi / \alpha, H_{0}(\alpha, M)\right)$. The proof is completed by applying the lemma to $\pi / \alpha$ and $H_{0}(\alpha, M)$.

Proposition 1.3. If $\pi$ is a finite nilpotent group and $M$ is a finite nilpotent $\pi$-module with $H_{n}(\pi, M)=0$ for some $n \geqslant 1$, then $M$ is cohomologically trivial.

Proof. It suffices to show that $H_{i}(\alpha, M)=0$ for any Sylow $p$-group $\alpha$ and for all $i \geqslant 1$. Using the "elementary facts" referred to in the proof above, the spectral sequence for $0 \rightarrow \alpha \rightarrow \pi \rightarrow \pi / \alpha \rightarrow 0$ yields for any $k$ a short exact sequence

(*) $\quad 0 \rightarrow H_{0}\left(\pi / \alpha, H_{k}(\alpha, M)\right) \rightarrow H_{k}(\pi, M) \rightarrow H_{k}\left(\pi / \alpha, H_{0}(\alpha, M)\right) \rightarrow 0$.

If $H_{n}(\pi, M)=0$, then $H_{n}(\alpha, M)$ is both perfect and nilpotent, hence 0. Apply Proposition 1.1 to complete the proof.

Next, we relax the condition that $M$ be a finite group. 
Proposition 1.4. Let $\pi$ be a finite nilpotent group and let $M$ be a nilpotent $\pi$-module. If $H_{n}(\pi, M)=H_{n+1}(\pi, M)=0$ for some $n \geqslant 1$, then $M$ is cohomologically trivial. Furthermore, if $M$ is finitely generated it must be finite (unless $\pi$ is 0 ).

Proof. Let $\alpha$ be a Sylow $p$-group of $\pi$. As in the preceding proof, derive the sequence (*). Because $M$ is nilpotent, the hypothesis forces $H_{n}(\alpha, M)=$ $H_{n+1}(\alpha, M)=0$. By Rim's Theorem 4.12 [16], $M$ is cohomologically trivial.

Suppose now that $M$ is finitely generated. Let $T$ denote the torsion submodule, a finite group. Let $\alpha$ be any cyclic subgroup of $\pi$. We know that $H_{1}(\alpha, M)=0$, and it is elementary that $H_{2}(\alpha, M / T)=0$ because $M / T$ must be $\pi$-trivial (see Lemma 1.5). Therefore $H_{1}(\alpha, T)=0$. By Proposition 1.1 all the homology of $T$ over $\alpha$ vanishes, which implies that $M / T$ must be 0 .

LEMMA 1.5. Let $P$ be a set of primes and suppose that $\pi$ is a P-group (not necessarily finite or nilpotent). If $M$ is a nilpotent $\pi$-module having no $P$-torsion, then it must be $\pi$-trivial.

Proof. Deny it. If $M$ is not $\pi$-trivial it must contain a submodule $N$ that is not $\pi$-trivial but $I N$ is $\pi$-trivial. Pick any $n \in N$ and $x \in \pi$. For any $k \geqslant 1$,

$$
x^{k-1}(x n-n)=x n-n,
$$

so $x^{k} n=x^{k-1} n+x n-n$. The solution of this recurrence equation is $x^{k} n=k(x n)$ $-(k-1) n$. There is some integer $r$, a product of primes in $P$, such that $x^{r}=1$. Thus, $r(x n-n)=0$, so that $x n=n$, contradicting the definition of $N$.

We wish to derive analogies of 1.1 and 1.2 for infinite $\pi$.

Proposition 1.6. Suppose $\pi$ is a finitely generated nilpotent group, not finite, and that $M$ is a nilpotent $\pi$-module (not necessarily finitely generated). Let $n$ denote the rank of $\pi$. Suppose $H_{k}(\pi, M)=0$ for some $k \geqslant 0$.

(1) If $k \leqslant n$, then $M=0$.

(2) If $k>n$, then $H_{j}(\pi, M)=0$ for all $j>n$.

Proof. The proof is by induction on $n$. Since $n \geqslant 1$ we can find a normal subgroup $\alpha$ of $\pi$ with quotient $Z$. From the Lyndon-Hochschild-Serre spectral sequence derive for any $j \geqslant 1$ the short exact sequence

$$
0 \rightarrow H_{0}\left(Z, H_{j}(\alpha, M)\right) \rightarrow H_{j}(\pi, M) \rightarrow H_{1}\left(Z, H_{j-1}(\alpha, M)\right) \rightarrow 0 .
$$

As in several earlier proofs, we conclude that $H_{k}(\alpha, M)=0$.

Recall that $H_{1}(Z, M)$ is the submodule of $M$ consisting of all the fixed elements of the action. For a nontrivial nilpotent module, this submodule cannot be 0 . Thus, $H_{k-1}(\alpha, M)$ must also be 0 .

To get the induction started, assume first that $n=1$. Case (1) of the theorem, then, assumes that $k \leqslant 1$. If $k=0$ then obviously $M$ must be 0 . If $k=1$, we have from the preceding paragraph that $H_{0}(\alpha, M)=0$, hence $M$ must be 0 here too. Case (2) of the theorem translates into " $k>1$ ". Knowing that $H_{k-1}(\alpha, M)=H_{k}(\alpha, M)$ $=0$ allows us to use Proposition 1.4 and deduce that $H_{j}(\alpha, M)=0$ for all $j \geqslant 1$. Thus $H_{j}(\pi, M)=0$ for all $j \geqslant 2$, as claimed. 
To establish the inductive step, assume that $n \geqslant 2$ and that the theorem is known for any finitely generated nilpotent group of rank less than $n$. The normal subgroup $\alpha$ obviously has rank $n-1$. The routine verification of cases (1) and (2) is left to the reader.

The final result of this type is a blend of 1.4 and 1.6. We would like to know what happened if $\pi$ is finite, $M$ is infinite, nilpotent, and has only one known vanishing homology group. It is certainly not true that the vanishing of any homology of $M$ implies the vanishing of all the others: take $M Z$-free with trivial action.

Proposition 1.7. Let $\pi$ be a finite nilpotent group, $M$ a finitely generated nilpotent $\pi$-module with $H_{1}(\pi, M)=0$. Then $M$ is cohomologically trivial and, unless $\pi$ is 0 , is $a$ finite group.

Proof. Suppose first that $\pi$ is cyclic. Exactly as in the proof of Proposition 1.4, deduce that the torsion submodule of $M$ has vanishing homology, forcing $H_{2}(\pi, M)$ to be 0 . The result follows by Proposition 1.4.

The proof for the general case follows now much as in the proof of Proposition 1.2 , by induction on $|\pi|$. The details are left to the reader.

Proposition 1.7 is false without the finite generation hypothesis on $M$. For example, let $M$ be the dyadic rationals modulo the integers, and think of $M$ as a trivial $Z_{2}$-module. The reader may verify that $H_{1}\left(Z_{2}, M\right)=0$ but $H_{2}\left(Z_{2}, M\right)=Z_{2}$.

This completes the proof of Theorem A.

Finally, we present one more result of a similar flavor that will be used in a later section.

Proposition 1.8. Let $\pi$ be a finite nilpotent group and $P$ a finitely generated $\pi$-module. If $P$ is $\pi$-perfect and cohomologically trivial, then $P$ is a finite group.

Proof. Let $T$ denote the torsion submodule of $P$ and $F$ the quotient $P / T$. Then $T$ and $F$ and both $\pi$-perfect.

If $\pi$ is a $p$-group, $F$ must be zero because a $p$-group cannot otherwise act perfectly on a $Z$-free module. We will show that $F$ must be 0 in general by induction on $|\pi|$.

Let $\alpha$ be any nontrivial normal subgroup of $\pi$ and consider $H^{0}(\alpha, P) \subset P$. This $\pi$-module must be $\pi$-perfect; hence is perfect as a $\pi / \alpha$-module. Let $\pi^{\prime}$ be any subgroup of $\pi$ containing $\alpha$ and consider the spectral sequence $H^{s}\left(\pi^{\prime} / \alpha, H^{t}(\alpha, P)\right)$ $\Rightarrow H^{s+t}\left(\pi^{\prime}, P\right)$. By assumption, $H^{t}(\alpha, P)=0=H^{t}\left(\pi^{\prime}, P\right)$ if $t>0$. It follows that $H^{0}(\alpha, P)$ is cohomologically trivial as a $\pi^{\prime} / \alpha$-module. By the inductive hypothesis it must be a finite group.

From the long exact sequence associated with $0 \rightarrow T \rightarrow P \rightarrow F \rightarrow 0$ we then deduce that $H^{0}(\alpha, F) \cong H^{1}(\alpha, T)$, which obviously forces $H^{0}(\alpha, F)=H^{1}(\alpha, T)=$ 0 . By Proposition $1.1 H_{i}(\alpha, T)=0 \forall i \geqslant 1$. As $P$ is cohomologically trivial, $H_{i}(\alpha, F)$ $=0 \forall i \geqslant 1$.

Now assume that $\alpha$ is a cyclic p-group. $H_{*}(\alpha, F)$ may be computed with the resolution $(0.1) . H^{0}(\alpha, F)=0$ implies that the $\alpha$ norm map $N: F \rightarrow F$ is 0 . Consequently $\operatorname{ker} N=\operatorname{im} T=F$, which means that $F$ is $\alpha$-perfect. This forces $F=0$ as desired.

Another theorem of this general type is Corollary 1.7 of [8]. 
2. The Euler characteristic of a nilpotent complex is zero. The precise statement is

Proposition 2.1. Let $\pi$ be a nontrivial finitely generated nilpotent group. Let $X$ be a nilpotent finitely dominated complex with fundamental group $\pi$. Then the Euler characteristic $\chi(X)=0$. If $\pi$ is finite, then $\chi(\tilde{X})=0$.

REMARK. For the case $\pi$ finite this result was stated in [12, proof of Lemma 3.4]. ${ }^{1}$

Proof. First assume that $\pi$ is finite. We claim that $\chi(X)=\chi(\tilde{X})$, because in fact $\operatorname{rank}_{Z} H_{*}(X)=\operatorname{rank}_{Z} H_{*}(\tilde{X})$. To see this, examine the Serre spectral sequence of the fibration $\tilde{X} \rightarrow X \rightarrow K(\pi, 1)$. Clearly $E_{s t}^{\infty}$ is a finite group if $s \geqslant 1$. Thus $\operatorname{rank}_{Z} H_{k}(X)=\operatorname{rank}_{Z} E_{0, k}^{\infty}$. But since $E_{s t}^{2}$ is finite for $s \geqslant 1, \operatorname{rank}_{Z} E_{0, k}^{\infty}=\operatorname{rank}_{Z} E_{0, k}^{2}$. It is well known that a nilpotent $Z$-free $\pi$-module must be $\pi$-trivial if $\pi$ is finite. When that fact is applied to the module obtained from $H_{k}(\tilde{X})$ by reducing modulo torsion, it follows that $\operatorname{rank}_{Z} H_{k}(\tilde{X})=\operatorname{rank}_{Z} E_{0, k}^{2}$. This establishes the claim.

Were $X$ a finite complex, the claim above combined with the obvious fact that $\chi(\tilde{X})=|\pi| \chi(X)$ would complete the proof. But by a theorem of $\mathrm{K}$. Brown [1, p. 231], the same equation $\chi(\tilde{X})=|\pi| \chi(X)$ holds for finitely dominated $X$. This completes the proof for the case $\pi$ finite (and $\neq 0$ ).

If $\pi$ is infinite, find a surjection $\pi \rightarrow Z$ and call the kernel $\alpha$. Consider the Serre spectral sequence of the fibration $\tilde{X}_{\alpha} \rightarrow X \rightarrow K(Z, 1)$. Clearly $E_{s t}^{2}=E_{s t}^{\infty}$, and $\operatorname{rank}_{Z} H_{k}(X)=\operatorname{rank}_{Z} E_{1, k-1}^{2}+\operatorname{rank}_{Z} E_{0, k}^{2}$. By considering the resolution (0.1), we see that $\operatorname{rank}_{Z} H_{0}(Z, M)=\operatorname{rank}_{Z} H_{1}(Z, M)$, where $M$ is any finitely generated (as a group) $Z$-module. Consequently, the alternating sum

$$
\chi(X)=\sum_{k}(-1)^{k} \operatorname{rank}_{Z} H_{0}\left(Z, H_{k} \tilde{X}_{\alpha}\right)+\sum_{k}(-1)^{k} \operatorname{rank}_{Z} H_{0}\left(Z, H_{k-1} \tilde{X}_{\alpha}\right)=0 .
$$

We shall discuss applications of Proposition 2.1 to the theory of finitely dominated nilpotent $\mathrm{CW}$ complexes, and, in a later section, to the question of the existence of homology decompositions of nilpotent complexes.

Basic references for the topic of finitely dominated nilpotent complexes are $[\mathbf{1 0}, 11$ and 14].

Let $K_{0}(\pi), \tilde{K}_{0}(\pi)$ and $G(\pi)$ have their conventional meanings. In [20] Swan defines the Grothendieck groups $P_{0}(\pi)$ and $C_{0}(\pi) . P_{0}(\pi)$ is generated by finitely generated projectives $P$ having the property that $P \otimes_{Z} Q$ is $Q \pi$-free. $C_{0}(\pi)$ is the kernel of the natural map $P_{0}(\pi) \rightarrow Z$ given by rank ${ }_{Q \pi}$. He shows [19] that for finite $\pi, P_{0}(\pi)$ is in fact all of $K_{0}(\pi)$. It follows that there is a natural isomorphism $\tilde{K}_{0}(\pi) \rightarrow C_{0}(\pi)$ given by $[P] \rightarrow[P]-[F]$, where $F$ is free of the same rank as $P$.

Now let $\mathcal{O}$ be a maximal order of $Q \pi$ containing $Z \pi$. We may likewise define $C_{0}(\mathcal{O})$ and $G(\mathcal{O})$. Tensoring over $Z \pi$ with $\mathcal{O}$ induces a map $C_{0}(\pi) \rightarrow C_{0}(\mathcal{O})$, and there is a forgetful map $G(\mathcal{O}) \rightarrow G(\pi)$. We may construct the following commutative diagram:

$$
\begin{array}{ccc}
C_{0}(\pi) & \rightarrow & G(\pi) \\
\text { epi } \downarrow \xi & \nearrow \psi & \uparrow \text { epi } \\
C_{0}(\mathcal{O}) & \underset{\text { mono }}{\rightarrow} & G(\mathcal{O})
\end{array}
$$

\footnotetext{
${ }^{1}$ I would like to thank G. Mislin for bringing this reference to my attention.
} 
Besides the indicated properties, true for any $\pi$, Swan proves that $\psi$ is a monomorphism if $\pi$ is a cyclic $p$-group. Further, $\xi$ is an isomorphism if $\pi=Z_{p}$ (Rim's Theorem).

Let $X$ be a finitely dominated nilpotent complex with finite fundamental group $\pi$. The reduced Wall obstruction $\tilde{w}(X)$ is an element of $C_{0}(\pi)$ given by $\Sigma(-1)^{i}\left[P_{i}\right]-$ $\sum(-1)^{i}\left[F_{i}\right]$, where the $\left\{P_{i}\right\}$ are a projective resolution of $Z$ chain homotopy equivalent to the chains of $\tilde{X}$, and $F_{i}$ is free of the same rank as $P_{i}$. By Proposition 2.1, $\sum(-1)^{i}\left[F_{i}\right]=0$.

COROllary 2.2. The image of $\tilde{w}(X)$ in $G(\pi)$ is 0 .

Proof. Note that in $G(\pi), \tilde{w}(X)=\sum(-1)^{i}\left[H_{i} \tilde{X}\right]$.

For each $i$ let $T_{i}$ denote the torsion submodule of $H_{i} \tilde{X}$ and $R_{i}$ the quotient $H_{i} \tilde{X} / T_{i}$. Since $R_{i}$ is nilpotent and $Z$-free, it is isomorphic to $\operatorname{rank}_{Z} H_{i} \tilde{X}$ copies of $Z$. We will show in the following lemma that $\left[T_{i}\right]=0$ in $G(\pi)$. So by Proposition 2.1, $\tilde{w}(X)=0$ in $G(\pi)$.

LEMMA 2.3. If.M is a finite nilpotent $\pi$-module, then $[M]=0$ in $G(\pi)$.

Proof. By induction on the nilpotency height, it suffices to prove the lemma for $\pi$-trivial finite modules. But such a module is the direct sum of cyclic groups $Z_{n}$, for which there is a sequence $0 \rightarrow Z \rightarrow Z \rightarrow Z_{n} \rightarrow 0$.

Corollary 2.2, and the results of Swan, provide very simple proofs of the major results of Mislin in [10]. For example, if $\pi$ is a cyclic $p$-group, the vanishing of $\tilde{w}(X)$ in $G(\pi)$ implies that it vanishes in $C_{0}(\mathcal{O})$. This means that $\tilde{w}(X)$ is in the group called $D(\pi)$. If $\pi$ is $Z_{p}, \xi$ is an isomorphism and so $\tilde{w}(X)$ must be 0 .

In [14] Mislin and Varadarajan establish the deep result that $\tilde{w}(X)$ vanishes in $G(\mathcal{O})$ for any finite $\pi$. We will briefly sketch a proof of this fact that mimics the proof of Corollary 2.2.

Note that in $G(\mathcal{O}), \tilde{w}(X)=\Sigma(-1)^{i}\left[H_{i}(X, \mathcal{O})\right]$, local coefficient homology. There is an exact sequence of $Z_{\pi}$ modules $0 \rightarrow Z_{\pi} \rightarrow \mathcal{O} \rightarrow T \rightarrow 0$, where $T$ is a finite group, and a corresponding long exact sequence of $\pi$-modules $\cdots \rightarrow H_{k} \tilde{X} \rightarrow H_{k}(X, \mathcal{O}) \rightarrow$ $H_{k}(X, T) \rightarrow \cdots$. (These groups are modules because $Z \pi$ and $\mathcal{O}$ are left-right bimodules.)

Since $T$ is finite, it is a prenilpotent $\pi$-module; let $P$ denote its perfect submodule and $N$ the nilpotent quotient. Since $X$ is nilpotent it is not hard to show that $H_{*}(X, P)=0$, so that $H_{*}(X, T)=H_{*}(X, N)$. It is also not hard to show that this last module is nilpotent. Referring to the long exact sequence above, we conclude that $H_{*}(X, \mathcal{O})$ is nilpotent over $Z \pi$.

As in the proof of Corollary 2.2, let $T_{i}$ denote the torsion submodule (as a $\pi$ - or O-module) of $H_{i}(X, \mathcal{O})$ and $R_{i}$ the quotient. As before, $\pi$ acts trivially on $R_{i}$. But since $\mathcal{O} / Z \pi$ is finite and $R_{i}$ is $Z$-free, $\mathcal{O}$ also acts trivially on $R_{i}$ (that is, via the augmentation map $\mathcal{O} \rightarrow Z$ ). Thus, just as in the earlier proof, the alternating sum $\sum(-1)^{i}\left[R_{i}\right]$ vanishes. We are left with the task of showing that a finite $\mathcal{O}$-module that is nilpotent over $Z \pi$ represents 0 in $G(\mathcal{O})$. This is proven via number theoretic techniques on pp. 188-190 of [14]. 
Our second application of Proposition 2.1 is the following result, which will be used in $\S 4$.

Corollary 2.4. Let $F \underset{i}{\rightarrow} \underset{p}{\rightarrow} B$ be a fibration of finitely dominated nilpotent complexes. Then $\tilde{w}(E)$ vanishes in either of the following cases:

(1) $\tilde{w}(B)=0, \pi_{1}(B) \neq 0$.

(2) $F$ is the universal cover of a finitely dominated complex with finite fundamental group.

Proof. We may assume that $\pi_{1}(E)$ is finite. The proof is based on K. Ehrlich's Theorem 3.5 [5]. In case (1) that theorem yields the formula $\tilde{w}(E)=\chi(B) \cdot i_{*} \tilde{w}(F)$, which obviously vanishes by Proposition 2.1.

In case (2) we have the formula $\tilde{w}(E)=\chi(p) \otimes p^{*} \tilde{w}(B)$, where $\chi(p) \equiv$ $\sum(-1)^{i}\left[H_{i} F\right] \in G\left(\pi_{1} E\right)$. This vanishes by Proposition 2.1 and Lemma 2.3.

3. Maps that are almost homology equivalences, and homology decompositions. It is by now classic that a homology equivalence between nilpotent complexes must be a homotopy equivalence. In this section we investigate maps between nilpotent complexes that fail to be homology equivalences in only one dimension, i.e., the cofiber of the map is a Moore space. For simply connected spaces this implies that the cofibration is induced: the larger space arises from the smaller by attaching the cone on a Moore space.

For nilpotent spaces we have

TheOREM B. Let $A \rightarrow X$ be a cofibration of nilpotent complexes with cofiber $X / A=K^{\prime}(G, n)$ for some group $G$ and some $n \geqslant 3$. Let $\pi=\pi_{1} X \neq 0$.

(1) If $\pi$ is infinite but finitely generated, then, $A \rightarrow X$ is in fact a homotopy equivalence ( $G$ must be 0 ).

(2) If $\pi$ is finite and $M \equiv H_{n}(\tilde{X}, \tilde{A})$ is a finitely generated $\pi$-module, then $\tilde{X} / \tilde{A}=$ $K^{\prime}(M, n) . M$ and $G$ must be finite, and $M$ must be cohomologically trivial. Furthermore, $X$ can be built from $A$ by attaching $n$ - and $(n+1)$-dimensional cells.

Proof. The assumption $n \geqslant 3$ guarantees that the map on $H_{1}$ is an isomorphism and on $H_{2}$ is an epimorphism. By Stalling's result [17], the induced map on $\pi_{1}$ is an isomorphism.

Consider the spectral sequence $E_{s t}^{2}=H_{s}\left(\pi, H_{t}(\tilde{X}, \tilde{A})\right) \Rightarrow H_{s+t}(X, A)$. Since $H_{2}(X, A)=0, H_{2}(\tilde{X}, \tilde{A})$ must be $\pi$-perfect, hence must be 0 . The same reasoning applied inductively shows that $(\tilde{X}, \tilde{A})$ is $(n-1)$-connected.

Now, $H_{n+1}(X, A)=0$ implies that $E_{1, n}^{\infty}=0$, which forces $E_{1, n}^{2}=0$. Hence $H_{1}\left(\pi, H_{n}(\tilde{X}, \tilde{A})\right)=0$. (1) is established immediately by Proposition 1.6.

As to (2), Proposition 1.7 tells us that $H_{n}(\tilde{X}, \tilde{A})$ is a finite cohomologically trivial module. But then the spectral sequence implies that $H_{n+1}(\tilde{X}, \tilde{A})$ is $\pi$-perfect, hence 0 . Inductively, $H_{k}(\tilde{X}, \tilde{A})=0$ for all $k>n$. So if $M$ denotes $H_{n}(\tilde{X}, \tilde{A})$, then $\tilde{X} / \tilde{A}=K^{\prime}(M, n)$.

We proceed to build $\tilde{X}$ out of $\tilde{A}$ by reattaching the relative cells in dimension $n$ and $n+1$. The technique is essentially the same used by Wall [21] and explained in 
Brown and Kahn [2]. Let $(\tilde{X}, \tilde{A})^{k}$ denote the $k$-skeleton of the relative complex. Since $M$ has a resolution over $\pi$ of length one, the kernel of $H_{n}(\tilde{X}, \tilde{A})^{n} \rightarrow M \rightarrow 0$ is projective, say $P$. Let $F$ be a free complement of $P$, say $F \oplus P=F^{\prime}$. Wedge on $n$-spheres equivariantly to $(\tilde{X}, \tilde{A})^{n}$, creating a complex $\tilde{Y}$ with $H_{n}(\tilde{Y}, \tilde{A})=$ $H_{n}(\tilde{X}, \tilde{A})^{n} \oplus F$. Examination of the exact sequences of the various pairs and triples shows that $(n+1)$-cells can be equivariantly attached to $\tilde{Y}$ so that the $\pi$-resolution $0 \rightarrow F^{\prime} \rightarrow F \oplus H_{n}(\tilde{X}, \tilde{A})^{n} \rightarrow M \rightarrow 0$ is realized by $0 \rightarrow H_{n+1}(\tilde{Y}, \tilde{A})^{n+1} \rightarrow$ $H_{n}(\tilde{Y}, \tilde{A})^{n} \rightarrow M \rightarrow 0$. A homology equivalence $\tilde{Y} \rightarrow \tilde{X}$ is easily constructed. $Y \equiv$ $\tilde{Y} / \pi$ is the desired rebuilt $X$.

We can say a little more about both cases of the theorem. In (2), it does not follow that the cofibration $A \rightarrow X$ is induced; examples to the contrary are given in $\S 3$ of [8]. It is shown there that any $\pi$-resolution of length one of any nilpotent cohomologically trivial module $M$ can be used to attach cells of dimension $n$ and $n+1$ to a nilpotent $A$, yielding a nilpotent $X$ with $X / A$ a Moore space. The cofibration is induced iff $M$ is an induced module.

As for (1), if $A \rightarrow X$ is not a homotopy equivalence but does induce an isomorphism on $\pi_{1}$, then the first nonvanishing homology groups of $(X, A)$ must come paired, one after another. Furthermore, if $(X, A)$ is finite dimensional, the last nonvanishing homology groups must also be consecutive. The proof is left to the reader.

In the simply-connected case, the fact that every cofibration with cofiber a Moore space is induced is the beginning of the theory of homology decompositions [7].

Given a simply-connected complex it is possible to find a homotopy equivalent normal complex $X$ having a decomposition into a chain of subcomplexes $X_{2} \subset X_{3} \subset$ ‥ such that $X_{n} / X_{n-1}=K^{\prime}\left(H_{n} X, n\right)$, and with each cofibration induced since all spaces are simply connected. Suprisingly, it is shown in [9] that the normal complex construction works for a large class of nonsimply-connected spaces (those that have finitely presented fundamental group), although there is no longer any reason for the cofibrations in the chain to be induced. Because the homotopy type of a nilpotent complex is determined by its homology, it has been conjectured that a nilpotent complex has a homotopy equivalent normal complex consisting of nilpotent $X_{n}$ 's, where the chain starts at $X_{2}$ (or possibly $X_{k}, k>2$ ), a nilpotent complex of low dimension and few homology groups.

THEOREM C. If a finitely dominated nilpotent complex can be decomposed into a normal chain of subcomplexes, then either it is simply connected or the decomposition is trivial.

Proof. Suppose that the fundamental group is infinite. By (1) of Theorem B, every $X_{n}=X_{n-1}$.

Suppose that the fundamental group is finite but not zero. Since three dimensional nilpotent complexes exist (see \$4), we may imagine starting the chain at $X_{2}$, as desired. But by Proposition 2.1, $H_{3} X_{2} \neq 0$, so the classical construction fails. Furthermore, by (2) of Theorem $\mathrm{B}$, only finite homology groups $H_{n} X$ could be added on this way. 
4. Nilpotent complexes of minimal dimension. In [2] Brown and Kahn established a lower bound on the dimension of a nilpotent complex having a given fundamental group $\pi$, and asked when this bound is attained. We show that it is always attained. However, if $\pi$ is finite the complex we construct (of dimension three) is not necessarily finite for arbitrary $\pi$. The question therefore arises, is there a finite complex of minimal dimension? We show that there is always a finite six-dimensional complex. Further, if $\pi$ is torsion free, the universal cover of any minimal dimension complex is an equivariant Moore space.

We begin with the statement of the lower bound.

THEOREM (BROWN AND KAHN). Let $\pi$ be a nontrivial finitely generated nilpotent group, and suppose that $X$ is an $n$-dimensional nilpotent complex with fundamental group $\pi$.

(1) If $\pi$ is finite, then $n \geqslant 3$.

(2) If $\pi$ is torsion free and $X \neq K(\pi, 1)$, then $n \geqslant \operatorname{rank}(\pi)+2$.

(3) If $\pi$ has torsion, then $n \geqslant \operatorname{rank}(\pi)+3$.

Proof. [2, Corollary 5.4].

THEOREM D. For any finitely generated nilpotent group $\pi$ the minimal dimension is realized by a finitely dominated nilpotent complex $X$. Further;

(1) If $\pi$ is torsion free, the universal cover $\tilde{X}$ is an equivariant Moore space of type $(M, 2, \pi)$, where $M$ is $Z$-free and nilpotent.

(2) If $\pi$ is infinite, $X$ may be taken finite.

(3) For the case $\pi$ finite, if $\pi$ is cyclic, or is a p-group, $X$ may be taken finite. For any finite $\pi$ there is a finite six-dimensional nilpotent complex.

The proof is organized as follows. We prove (1) first because it is easy. Then we prove (2) because it is easy modulo (3). This leaves us with the proof of (3), wherein lies the difficulty.

Proof of Theorem D(1). Let $X=K(\pi, 1) \times S^{2} . \tilde{X}=\tilde{K}(\pi, 1) \times S^{2}$ and the action on $H_{2} \tilde{X}=Z$ is trivial, so $X$ is nilpotent and finite. On the other hand, if $X$ is any such complex, then $\tilde{X}$ must have homotopy dimension at most two (by [2, proof of Lemma 2.3]), so $H_{2} \tilde{X}$ must be $Z$-free and $H_{i} \tilde{X}=0, i \neq 2$.

REMARK. By Theorem 3.1 of [8], there exists a free-based equivariant Moore space of type $\left(H_{2} \tilde{X}, 2, \pi\right)$. Carlsson has proven [3] that for many triples $(M, n, \pi)$ no equivariant Moore space exists. It would therefore be interesting to see examples of such $\pi$ and $X$ in which $\mathrm{H}_{2} \tilde{X}$ is not $\pi$-trivial.

Proof of Theorem $\mathrm{D}(2)$. Let $r=\operatorname{rank}(\pi)$, let $\alpha$ be a torsion free normal subgroup of $\pi$ of rank $r$ and let $X^{3}$ be a three-dimensional nilpotent complex with $\pi_{1} X^{3}=\pi / \alpha($ from Theorem $\mathrm{D}(3)$ ).

Consider the diagram

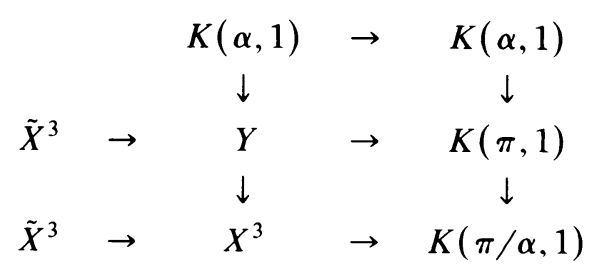


where $X^{3} \rightarrow K(\pi / \alpha, 1)$ and $K(\pi, 1) \rightarrow K(\pi / \alpha, 1)$ are the obvious fibrations, and $Y$ is the pullback. It is clear from the fibration $\tilde{X}^{3} \rightarrow Y \rightarrow K(\pi, 1)$ that $Y$ is nilpotent, and it is clear from the Serre spectral sequence of $K(\alpha, 1) \rightarrow Y \rightarrow X^{3}$ that $Y$ is $r+3$ dimensional (cf. [2, Theorem 5.1]). By Theorem B of [11], $Y$ has the homotopy type of a finite complex.

Proof of Theorem D(3). If $\pi$ is cyclic, let $X^{3}$ be the 3 -skeleton of a $K(\pi, 1)$ realizing the resolution (0.1). Then $H_{3} \tilde{X}^{3}=N \cdot Z \pi$, a nilpotent $\pi$-module.

In the general case, let $Y$ be the 2-skeleton of a $K(\pi, 1)$. The chains of $\tilde{Y}$ give an exact sequence $0 \rightarrow K \rightarrow F_{2} \rightarrow F_{1} \rightarrow Z \pi \rightarrow Z \rightarrow 0$, in which each $F_{i}$ is a finitely generated free module. This sequence is $Z$-split. Let $S$ denote the subring of $Q$ obtained from $Z$ by adjoining the inverse of each prime dividing $|\pi|$. Tensoring with $S$ yields $0 \rightarrow K^{\prime} \rightarrow F_{2}^{\prime} \rightarrow F_{1}^{\prime} \rightarrow S \pi \rightarrow S \rightarrow 0$. Because this sequence is split over $S$, we may (by "averaging") find an $S \pi$-splitting. It follows that there is an exact sequence $0 \rightarrow S \rightarrow F^{\prime} \underset{p^{\prime}}{\rightarrow} K^{\prime} \rightarrow 0$, in which $F^{\prime}$ is $S \pi$-free, of dimension $n$, say.

Now, if $F \equiv(Z \pi)^{n}$, there are canonical maps $F \rightarrow F^{\prime}$ and $K \rightarrow K^{\prime}$. We desire a map $p: F \rightarrow K$ lifting $p^{\prime}$. There may be no such lift, but there will be if $p^{\prime}$ is multiplied by a suitable integer, a product of primes dividing $|\pi|$. Hence the diagram

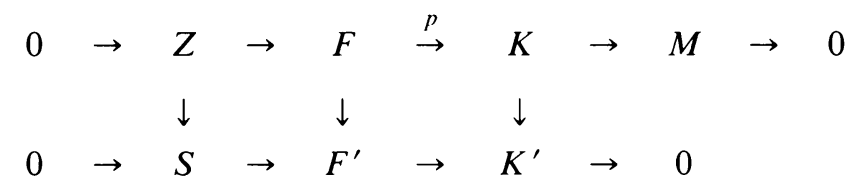

where the kernel of $p$ has no choice but to be $Z$ (with trivial $\pi$-action), and the cokernel $M$ is a finite module consisting of $|\pi|$ torsion.

The map $p$ can be realized geometrically. Since $K=H_{2} \tilde{Y}=\pi_{2} \tilde{Y}$, it is routine to equivariantly attach $n$ 3-cells, forming a complex $\tilde{W}^{3}$ such that $H_{3} \tilde{W}^{3}=Z$ and $H_{2} \tilde{W}^{3}=M$. If $\pi$ is a $p$-group then so is $M$. Since a $p$-group can only act nilpotently on a $p$-group, the proof is completed for that case.

In general, $M$ may have a perfect submodule $R$, so that $W^{3}$ is prenilpotent but not necessarily nilpotent. By Proposition 4.1, $R$ is cohomologically trivial. We can therefore apply the cell attaching method of $[8, \S 3]$ to construct a 4-dimensional nilpotent complex homology equivalent to $W^{3}$. Use a free $\pi$-resolution $0 \rightarrow F_{1} \rightarrow F_{0}$ $\rightarrow R \rightarrow 0$ [note: these modules may not be finitely generated] to attach 3- and 4-cells to $\tilde{W}^{3}$. The resulting complex $\tilde{V}^{4}$ satisfies $H_{4}\left(\tilde{V}^{4}, \tilde{V}^{3}\right)=F_{1}, H_{3}\left(\tilde{V}^{3}, \tilde{W}^{3}\right)=F_{0}$, $H_{4} \tilde{V}^{4}=0, H_{3} \tilde{V}^{4}=H_{3} \tilde{W}^{3}=Z$ and $H_{2} \tilde{V}^{4}=M / R$, a nilpotent module.

$V^{4}$ has the homotopy type of a 3-dimensional complex $X^{3}$, constructed as follows. By Theorem $5.1^{\prime}$ of $[8], P \equiv H_{3}\left(\tilde{V}^{4}, \tilde{W}^{2}\right)$ is projective. Furthermore, the homology sequence of the triple $\left(\tilde{V}^{4}, \tilde{W}^{3}, \tilde{W}^{2}\right)$ reduces to $0 \rightarrow F \rightarrow P \rightarrow R \rightarrow 0$ (where $F=$ $H_{3}\left(\tilde{W}^{3}, \tilde{W}^{2}\right)$ is the free module constructed earlier). The proof of Theorem 5.1' of [8] shows how to construct a complex $X^{3}$ homotopy equivalent to $V^{4}$, using a free complement of $P$, i.e., a free module $E$ such that $P \oplus E$ is free. $X^{3}$ is a finite complex only if $E$ is finitely generated. Indeed, it is clear that the Wall obstruction $\tilde{w}\left(X^{3}\right)=-[R]=[F]-[P]$ in $C_{0}(\pi)$.

This completes the existence proof for three-dimensional complexes. 
We claim now that a finite six-dimensional nilpotent complex can be found for any finite $\pi$. Let $X$ be the compiex constructed above and consider the following pullback diagram:

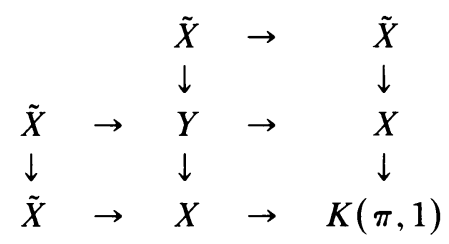

The desired complex is $Y$, by (2) of Corollary 2.4.

The following result was promised above.

Proposition 4.1. Let $X$ be a finite prenilpotent $n$-dimensional complex with finite fundamental group $\pi$. Suppose that every homology module $H_{*} \tilde{X}$ is nilpotent except $H_{n} \tilde{X}$ and $H_{n-1} \tilde{X}$. Let $P$ denote the perfect submodule of $H_{n-1} \tilde{X}$. The following are equivalent:

(1) $H_{n} \tilde{X}$ is nilpotent.

(2) $P$ is cohomologically trivial.

(3) $P$ is finite.

Proof. (2) $\Rightarrow$ (3) By Proposition 1.8.

$(1) \Rightarrow(2)$ Using the constructions of $[8, \S \S 4,5]$, we may build a nilpotent $n$-dimensional complex $Y$ and a homology equivalence $X \rightarrow Y$. The long exact homology sequence of the pair $(\tilde{Y}, \tilde{X})$ reduces to $0 \rightarrow P \rightarrow H_{n-1} \tilde{X} \rightarrow H_{n-1} \tilde{Y} \rightarrow 0$. Let $\alpha$ be a normal subgroup of $\pi$ and consider the spectral sequence of the fibration pair $E_{s t}^{2}=H_{s}\left(\alpha, H_{t}(\tilde{Y}, \tilde{X})\right) \Rightarrow H_{s+t}\left(\tilde{Y}_{\alpha}, \tilde{X}_{\alpha}\right)$. Every row $E_{*, t}^{2}$ is 0 except for $t=n$. Since $\tilde{Y}_{\alpha}$ and $\tilde{X}_{\alpha}$ are $n$-dimensional complexes, $E_{s n}^{\infty}=E_{s n}^{2}$ vanishes for $s$ large enough. This completes the proof by Theorem 4.12 of [16].

(3) $\Rightarrow$ (1) In notation of the previous case, the long exact homology sequence now reduces to $0 \rightarrow H_{n+1}(\tilde{Y}, \tilde{X}) \rightarrow H_{n} \tilde{X} \rightarrow H_{n} \tilde{Y} \rightarrow 0$ and $0 \rightarrow H_{n}(\tilde{Y}, \tilde{X}) \rightarrow H_{n-1} \tilde{X} \rightarrow$ $H_{n-1} \tilde{Y} \rightarrow 0$, with $H_{n+1}(\tilde{Y}, \tilde{X}) \pi$-perfect and $Z$-free. We would be done if $\pi$ were a p-group. The proof in general is by induction on the number of Sylow subgroups of $\pi$.

If $\alpha$ is any normal subgroup of $\pi$, the pair $\left(\tilde{Y}_{\alpha}, \tilde{X}_{\alpha}\right)$ has homology only in dimensions $n$ and $n+1$. Also, since $P=H_{n}(\tilde{Y}, \tilde{X})$ is finite, $H_{0}(\alpha, P)=H_{n}\left(\tilde{Y}_{\alpha}, \tilde{X}_{\alpha}\right)$ is finite.

Let $\alpha$ be the product of all Sylow subgroups but one, and consider the spectral sequence $E_{s t}^{2}=H_{s}\left(\pi / \alpha, H_{t}\left(\tilde{Y}_{\alpha}, \tilde{X}_{\alpha}\right)\right) \Rightarrow H_{s+t}(Y, X)$. As $H_{n+1}(Y, X)=0, E_{1, n}^{\infty}=$ $E_{1, n}^{2}=0$. By Proposition $1.1 E_{s n}^{2}=0 \forall s \geqslant 1$. Hence $E_{0, n+1}^{\infty}=E_{0, n+1}^{2}=0$, which means that $H_{n+1}\left(\tilde{Y}_{\alpha}, \tilde{X}_{\alpha}\right)$ is $Z$-free and $\pi / \alpha$-perfect, which means that it is 0 .

The induction argument consists of iterating the previous paragraph. In place of $\pi$ write $\alpha$, and in place of $\alpha$ put a $\beta$ such that $\alpha / \beta$ is a Sylow subgroup of $\pi$. In this way we eventually deduce that $H_{n+1}(\tilde{Y}, \tilde{X})=0$.

Theorem D provides new examples of nilpotent complexes with nonvanishing Wall obstruction, and of small dimension. The idea is a special case of the cell 
attaching methods of $[8, \S 3]$, and is a little more general than the technique of $[13, p$. 203].

EXAMPLE 4.2. Start with a finite $\pi$ and a finite nilpotent complex $X$, which we know can be of dimension as low as three or six. Find a nilpotent cohomologically trivial $\pi$-module $M$ such that $[M]$ in $C_{0}(\pi)$ is not zero. (This may not be possible for every $\pi$, but is for some; see, for example, Lemma 1.3 of [13].) Find a free resolution $0 \rightarrow F_{1} \rightarrow F_{0} \rightarrow M \rightarrow 0$, pick any $n \geqslant 2$ and use the resolution to attach $n$ - and $(n+1)$-dimensional cells to $\tilde{X}$ to form a complex $\tilde{Y}$ with $H_{n} \tilde{Y}=H_{n} \tilde{X} \oplus M$ and $H_{i} \tilde{Y}=H_{i} \tilde{X}$ for all $i \neq n$. Clearly, $\tilde{w}(Y)=(-1)^{n}[M]$.

\section{REFERENCES}

1. K. S. Brown, Euler characteristics of discrete groups and G-spaces, Invent. Math. 27 (1974), 229-264.

2. K. S. Brown and P. K. Kahn, Homotopy dimension and simple cohomological dimension of spaces, Comment. Math. Helv. 52 (1977), 111-127.

3. G. Carlsson, $A$ counterexample to a conjecture of Steenrod, Invent. Math. 64 (1981), 171-174.

4. W. Dwyer, Vanishing homology over nilpotent groups, Proc. Amer. Math. Soc. 49 (1975), 259-261.

5. K. Ehrlich, The obstruction to the finiteness of the total space of a fibration, Michigan Math. J. 28 (1981), 19-38.

6. K. Hoechsmann, P. Roquette and H. Zassenhaus, $A$ cohomological characterization of finite nilpotent groups, Arch. Math. (Basel) 19 (1968), 225-244.

7. P. J. Hilton, Homotopy theory and duality, Gordon \& Breach, New York, 1966.

8. R. H. Lewis, Equivariant cofibrations and nilpotency, Trans. Amer. Math. Soc. 267 (1981), 139-155.

9. __ Ph. D. dissertation, Cornell University, 1977.

10. G. Mislin, Wall's obstruction for nilpotent spaces, Topology 14 (1975), 311-317.

11. F_ Finitely dominated nilpotent spaces, Ann. of Math. (2) 103 (1976), 547-556.

12. __ Groups with cyclic Sylow subgroups..., Comment. Math. Helv. 52 (1977), 373-391.

13. The geometric realization of Wall obstructions.... Math. Proc. Cambridge Philos. Soc. 87 (1980), 199-206.

14. G. Mislin and K. Varadarajan. The finiteness obstructions for nilpotent spaces lie in $D(Z \pi)$, Invent. Math. 53 (1979), 185-191

15. D. J. S. Robinson, The vanishing of certain homology and cohomology groups, J. Pure Appl. Algebra 7 (1976), 145-167.

16. D. S. Rim. Modules over finite groups, Ann. of Math. (2) 69 (1959), 700-712.

17. J. Stallings, Homologv and central series of groups, J. Algebra 2 (1965), 170-181.

18. R. Swan, Periodic resolutions for finite groups, Ann. of Math. (2) 72 (1960), 267-291.

19. Induced representations and projective modules, Ann. of Math. (2) 71 (1960), 552-578

20. The Grothendieck ring of a finite group, Topology 2 (1963), 85-110.

21. C. T. C. Wall, Finiteness conditions for CW-complexes, Ann. of Math. (2) 81 (1965), 56-69.

Department of Mathematics, Fordham University, Bronx, New York 10458 